\title{
Regulation of genes affecting body size and innate immunity by the DBL-1/BMP-like pathway in Caenorhabditis elegans
}

\author{
Andrew F Roberts ${ }^{1,2+}$, Tina L Gumienny ${ }^{1,3+}$, Ryan J Gleason ${ }^{1}$, Huang Wang ${ }^{1}$, Richard W Padgett ${ }^{1 *}$
}

\begin{abstract}
Background: Bone morphogenetic proteins (BMPs) are members of the conserved transforming growth factor $\beta$ (TGF $\beta$ superfamily, and play many developmental and homeostatic roles. In C. elegans, a BMP-like pathway, the DBL-1 pathway, controls body size and is involved in innate immunity. How these functions are carried out, though, and what most of the downstream targets of this pathway are, remain unknown.

Results: We performed a microarray analysis and compared expression profiles of animals lacking the SMA-6 DBL-1 receptor, which decreases pathway signaling, with animals that overexpress DBL-1 ligand, which increases pathway signaling. Consistent with a role for DBL-1 in control of body size, we find positive regulation by DBL-1 of genes involved in physical structure, protein synthesis and degradation, and metabolism. However, cell cycle genes were mostly absent from our results. We also identified genes in a hedgehog-related pathway, which may comprise a secondary signaling pathway downstream of DBL-1 that controls body size. In addition, DBL-1 signaling upregulates pro-innate immunity genes. We identified a reporter for DBL-1 signaling, which is normally repressed but is up-regulated when DBL-1 signaling is reduced.

Conclusions: Our results indicate that body size in C. elegans is controlled in part by regulation of metabolic processes as well as protein synthesis and degradation. This supports the growing body of evidence that suggests cell size is linked to metabolism. Furthermore, this study discovered a possible role for hedgehog-related pathways in transmitting the BMP-like signal from the hypodermis, where the core DBL-1 pathway components are required, to other tissues in the animal. We also identified the up-regulation of genes involved in innate immunity, clarifying the role of DBL-1 in innate immunity. One of the highly regulated genes is expressed at very low levels in wildtype animals, but is strongly up-regulated in Sma/Mab mutants, making it a useful reporter for DBL-1/BMP-like signaling in C. elegans.
\end{abstract}

\section{Background}

Diverse cellular responses to TGF $\beta$ superfamily members are a hallmark of this family, with responses specified by cell type, time, or location within a TGF $\beta$ member gradient $[1,2]$. In C. elegans, a BMP-like family member, DBL-1 (Dpp and BMP-like), regulates not only body size, but also innate immunity and aspects of male tail development. Animals with reduced pathway signaling are small, while increased signaling results in long

\footnotetext{
* Correspondence: padgett@waksman.rutgers.edu

† Contributed equally

${ }^{1}$ Waksman Institute, Department of Molecular Biology and Biochemistry, Cancer Institute of New Jersey, Rutgers University, Piscataway, NJ 088548020, USA
}

(C) 2010 Roberts et al; licensee BioMed Central Ltd. This is an Open Access article distributed under the terms of the Creative Commons Attribution License (http://creativecommons.org/licenses/by/2.0), which permits unrestricted use, distribution, and reproduction in any medium, provided the original work is properly cited. more susceptible to bacterial or fungal infection, and DBL-1 is highly up-regulated upon infection $[5,6]$.

Body size and male tail development are separable by dose, as a weak sma-6 receptor mutant or a weak sma4/SMAD mutant affects body size but not male tails [7]. Furthermore, TGF $\beta$ pathway regulators also differentiate between body size and male tail development. For instance, sma-11/kin-29, bra-1, and lon-2/glypican affect body size but not male tails. MAB-23/DOUBLESEX transcription factor, on the other hand, affects DBL-1 male tail development independently of body size [8]. The LIN-31 forkhead transcription factor may also play a role in DBL-1 mediated male tail development. lin-31 
mutant males have crumpled spicules similar to $d b l-1$ mutant males, and forkhead transcription factors are known to be Smad co-factors $[9,10]$.

How is specificity achieved? We performed a microarray experiment comparing populations of mRNAs from animals with increased or decreased DBL-1/BMP signaling. We discovered that transcriptional control of body size in C. elegans acts through the regulation of metabolism, protein synthesis/degradation, and structural genes, and not obviously by cell cycle genes. Furthermore, we have identified a subset of the hedgehog-related genes (warthogs) as targets of the DBL-1 pathway, and propose that they act as downstream transducers of DBL-1 signaling for body size determination. In addition, we now better understand the role that DBL-1 plays in innate immunity, as our results show that genes known to be involved in innate immunity, namely lysozymes, lipase, and lectins, are regulated by the DBL-1 signaling pathway. A large number of other intestinally expressed genes, a primary site of innate immunity, are also highly regulated by DBL-1, suggesting a broader role for DBL1 in the intestinal innate immune response. Finally, we created a fluorescent biomarker for DBL-1 activity, and showed that the reporter accurately identified known DBL-1 signaling components.

\section{Results and Discussion}

\section{Microarray hybridization and analyses of gene expression profiles}

We compared gene expression in C. elegans strain BW1940 overexpressing ctIs40, an integrated transgene carrying wild-type $d b l-1$, and a strain lacking functional DBL-1 Type I receptor, LT186 sma-6(wk7) [4,7]. sma-6(wk7), which encodes a stop codon at Y72 predicted to truncate the protein prematurely in its extracellular domain, has reduced sma-6 RNA expression levels [7]. BW1940 animals are longer than the wild type, and LT186 animals are smaller than normal. These strains have not been tested for response to an immune challenge, however $d b l-1(n k 3)$ animals are more susceptible to infection by pathogenic bacteria [5,11-13].

We performed our microarray analyses with the Affymetrix C. elegans whole genome GeneChip array, which represents over 22,000 unique transcripts (Affymetrix, CA, \#900383). Five independent experiments were averaged and analyzed. About 2400 genes show a change in expression at the $95 \%$ confidence level $(<12 \%$ of total transcripts in the array), with about 1800 transcripts showing up-regulation of transcription in response to BMP signaling (positive regulation) and about 800 showing a down-regulation of transcription (negative regulation). 276 genes are regulated within a $99.9 \%$ confidence interval, with 186 positively regulated and 90 negatively regulated genes $(1.2 \%$ of total unique genes in the array)(Additional file 1). None of the genes in the 99.9\% confidence interval show a change in expression less than 1.5-fold (Additional file 2).

From our microarray results, we find that BW1940 ctIs $40(d b l-1(+))$ has about twice the amount of $d b l-1$ transcript as LT186 sma-6(wk7), which is consistent with it being overexpressed (Table 1). To validate the results of the microarray experiment, we performed qPCR on 27 genes that were highly up-regulated or down-regulated in our microarray analyses. We compared the ratio of expression of the two experimental genotypes in the qPCR and the microarray experiments to determine if the difference in levels showed the same trend. All but two samples showed the same trend (Table 1).

Table 1 Quantitative PCR results.

\begin{tabular}{|c|c|c|c|}
\hline Gene name & qPCR & Microarray & Agreement \\
\hline$d b /-1$ & & 2.12 & \\
\hline sma-6 & & 4.94 & \\
\hline K07C6.3 & 0.79 & 0.41 & YES \\
\hline $\mathrm{H} 12 \mathrm{l} 13.4$ & 0.80 & 0.80 & YES \\
\hline C25D7.6 & 0.91 & 0.28 & YES \\
\hline Y69H2.9 & 0.19 & 0.31 & YES \\
\hline C $42 C 1.8$ & 0.37 & 0.40 & YES \\
\hline T09F5.9 & 5.62 & 6.78 & YES \\
\hline F11A6.2 & 6.79 & 10.12 & YES \\
\hline Y19D10A.7 & 5.22 & 55.30 & YES \\
\hline $\mathrm{T} 10 \mathrm{H} 10.2$ & 0.24 & 0.16 & YES \\
\hline K02E2.8 & 2.12 & 52.80 & YES \\
\hline C29F3.2 & 5.77 & 1.48 & YES \\
\hline C29F3.5 & 9.96 & 5.095 & YES \\
\hline C05A9.1 & 1.88 & 3.77 & YES \\
\hline W09B7.2 & 5.90 & 6.42 & YES \\
\hline R02E12.6 & 0.72 & 0.33 & YES \\
\hline F44A2.1 & 0.13 & 0.12 & YES \\
\hline F01G10.3 & 0.33 & 0.25 & YES \\
\hline F21F8.4 & 0.16 & 0.44 & YES \\
\hline T21E8.1 & 8.49 & 25.64 & YES \\
\hline Y38E10A.5 & 4.21 & 12.19 & YES \\
\hline F56A4.2 & 13.65 & 11.45 & YES \\
\hline F35C5.9 & 5.92 & 5.88 & YES \\
\hline F55G11.4 & 15.78 & 5.79 & YES \\
\hline T11F9.4 & 2.60 & 3.72 & YES \\
\hline F59A7.2 & 0.88 & 0.40 & YES \\
\hline F55B12.4 & 1.23 & 0.27 & $\mathrm{NO}$ \\
\hline F15E11.10 & 1.02 & 9.57 & NO \\
\hline
\end{tabular}

The numeric values shown under each genotype tested represent the relative difference of BW1940 to LT186, with each value normalized to an internal standard. A value of 1 represents identical expression to the standard. 


\section{Regulation of body size genes}

How cell and organismal size is controlled is an old question that has been studied at the molecular level in yeast and only sporadically in multicellular organisms [14-16]. Body size is defined at the cellular level by cell number (a result of proliferation and cell death) and cell size $[14,17,18]$. Besides environmental factors and hormones, TGF $\beta$ superfamily signaling pathways have also been clearly implicated in controlling cell and body size in C. elegans, D. melanogaster [19-21], and in vertebrates $[22,23]$. Furthermore, because of TGF 3 superfamily pathways' roles in cell growth and proliferation, they are commonly associated with uncontrolled cell growth in cancers [24].

This study addresses the mechanisms by which body size is executed in a multicellular organism. C. elegans is the only model multicellular organism where the cell number is defined: 959 somatic cells in adult hermaphrodites and 1021 somatic cells in adult males [25]. By removing the cell number variable, our results focus on how cell size differences are achieved through our BMPlike signaling pathway.

Metabolic genes were enriched in our panel of highly up-regulated genes, including energy generation, protein expression, nucleotide synthesis, carbohydrate metabolism, amino acid metabolism and biosynthesis (Table 2). Additionally, we observed a small but consistent up-regulation of ribosomal proteins. Ribosomal proteins have been shown experimentally to be important for cell size regulation in yeast [26], Drosophila [27,28], and Arabidopsis [29]. Protein synthesis and degradation genes were also enriched (Additional file 3), including ubiquitinylation pathway proteins, suggesting that not only are increased amounts of protein required in the longer animal, but also increased protein turnover machinery.

Structural genes are also up-regulated by DBL-1 signaling (Additional file 4). Many non-dauer specific collagens and other extracellular matrix genes have increased gene expression at the 95th percentile with increased DBL-1 signaling. Intracellular structural genes, like actins, myosins, and tubulins also show positive changes. However, whether these drive body size changes or are a response to the need for more structural proteins by larger cells remains uncertain.

Germline genes comprise the largest category of genes down-regulated by DBL-1 signaling in our data set. These categories include mitotic and meiotic genes as well as DNA repair genes and oocyte specific genes (Table 2). Recently, the DBL-1 signaling pathway was shown to negatively regulate reproductive aging [30]. Pathway mutants appear to extend the reproductive span of older hermaphrodites by improving oocyte quality, not by affecting ovulation rate, early progeny number, or brood size. The model proposed is that DBL-1 normally modulates reproductive rates in response to environmental stress, and that loss of DBL-1 constitutively extends reproductive aging. Somatic life span was largely independent from germline health span. Our results indicate that the mechanism by which this phenomenon acts is through transcriptional regulation of germline-specific genes. We tested if altered regulation of germline genes affected brood size. To test this idea, we picked single $\mathrm{L} 4$ animals to plates and allowed them to lay eggs. The parental hermaphrodites were transferred and the eggs were counted every eight hours until no more eggs were laid. DBL-1 overexpressing animals (BW1940) had an identical brood size (272 eggs on average, $\mathrm{n}=10)$ to wild type. sma- 6 animals show a significantly smaller brood size ( $\mathrm{p}=.002)$, averaging only about 122 eggs. This brood size is similar to those of other mutant strains that have loss of DBL-1 pathway gene function [30]. Furthermore, eggs and embryos from mutants in the DBL-1 pathway are of normal size and the gonad from DBL-1 overexpressing animals is not proportionally bigger (our unpublished observations and [31]). Our results suggest that increased DBL-1 pathway signaling does not greatly affect the germline but loss of signaling does, by increasing expression of normally repressed germline-specific genes.

Cell cycle genes appear to be largely unaffected at the transcriptional level by DBL-1 signaling at the L4 stage. Other TGF $\beta$ superfamily members have been implicated in cell cycle regulation and cell proliferation, not only during development and homeostasis, but also during cancer progression [24,32]. In C. elegans, body size is dissociated from cell proliferation and number; however it is associated with endoreduplication in the polyploid hypodermal cells. Long animals with increased DBL-1 signaling have increased ploidy in hypodermal cells, and small animals with decreased DBL-1 signaling have reduced ploidy at later stages $[3,31,33,34]$. This indicates that some cell cycle genes are regulated by DBL-1, perhaps post-transcriptionally or at a level that does not reach significance in our analyses. Further, cell cycle genes may be altered at later stages of development. DBL-1 signaling does not affect the organism's maturation time or number of somatic cells, but pathway mutants do have reduced brood sizes, as indicated above. This could be an indication of cell cycle regulation in the adult gonad $[4,7,35]$.

Another similar but distinct published analysis has produced overlapping results. Mochii et al. (1999) screened an arrayed filter of C. elegans cDNAs (representing 7584 genes) for differences in regulation between $d b l-1(l f)$, sma-2(lf), lon-2(lf), and wild-type populations of third larval-stage animals [36]. Their results showed 20 genes ( 22 clones) that were both highly down-regulated in $d b l-1(l f)$ and sma-2(lf) animals 
Table 2 Microarray results associated with coregulated gene groups.

\begin{tabular}{|c|c|c|c|c|}
\hline Gene List & Representation Factor & $\mathrm{P}$-value & Regulated genes & \# in group \\
\hline \multicolumn{5}{|c|}{ Up-regulated } \\
\hline Mount 8 & 1.9 & $<1.6 \mathrm{e}-14$ & 153 & 803 \\
\hline Mount 20 & 1.6 & $<0.007$ & 27 & 160 \\
\hline Mount 23 & 4.6 & $<4.1 \mathrm{e}-29$ & 67 & 143 \\
\hline Mount 24 & 2 & $<1.8 \mathrm{e}-04$ & 28 & 133 \\
\hline Mount 27 & 4.8 & $<2.9 \mathrm{e}-20$ & 43 & 87 \\
\hline Mount 30 & 1.9 & $<0.071$ & 7 & 36 \\
\hline Mount 31 & 4.3 & $<1.5 \mathrm{e}-05$ & 11 & 25 \\
\hline Amino Acid Metabolism & 1.3 & $<0.179$ & 14 & 104 \\
\hline Biosynthesis & 1.3 & $<0.012$ & 65 & 478 \\
\hline Carbohydrate Metabolism & 1.5 & $<0.040$ & 19 & 121 \\
\hline Cell Structure & 1.4 & $<0.042$ & 31 & 219 \\
\hline Cell biology & 1.8 & $<0.077$ & 8 & 44 \\
\hline Collagen & 1.5 & $<0.16$ & 28 & 179 \\
\hline Energy Generation & 2.1 & $<3.0 \mathrm{e}-04$ & 25 & 117 \\
\hline Intestine & 3.6 & $<0.041$ & 3 & 8 \\
\hline Nucleotide Synthesis & 2.2 & $<0.004$ & 14 & 62 \\
\hline Proteases & 2.1 & $<2.6 \mathrm{e}-04$ & 25 & 116 \\
\hline Protein Expression & 2.2 & $<9.9 \mathrm{e}-14$ & 90 & 390 \\
\hline RNA binding & 2.7 & $<2.6 \mathrm{e}-13$ & 59 & 209 \\
\hline \multicolumn{5}{|c|}{ Down-regulated } \\
\hline Mount 7 & 3.1 & $<2.3 e-28$ & 115 & 810 \\
\hline Mount 11 & 3.3 & $<1.2 \mathrm{e}-24$ & 90 & 587 \\
\hline DNA Repair Genes & 5.5 & $<3.3 \mathrm{e}-06$ & 11 & 44 \\
\hline Germ Line Enriched & 3.6 & $<1.2 \mathrm{e}-24$ & 83 & 508 \\
\hline Meiosis & 3.8 & $<0.019$ & 4 & 23 \\
\hline Mitosis & 2.7 & $<0.003$ & 10 & 80 \\
\hline Oocyte-enriched & 1.8 & $<0.008$ & 21 & 258 \\
\hline
\end{tabular}

Specific functional groups were found to be over-represented in our $99.0 \%$ significant group using Stanford Microarray Database web tools [57]. A representation factor of 1.0 would be expected in a randomly generated list of genes. Higher values show enrichment for genes in that functional group. $P$ values represent the likelihood of achieving that enrichment by chance. Mount 7 contains germline enriched, oocyte, mitosis, and meiosis genes, Mount 8 contains intestinal genes, proteases, carboxylesterases, lipases, and antibacterial proteins. Mount 11 contains germline enriched, oocyte, meiosis, mitosis, retinoblastoma enriched complex. Mount 20 contains germline enriched, biosynthesis, protein expression, and heat shock genes. Mount 23 contains protein expression and energy generation genes. Mount 24 contains amino acid metabolism, lipid metabolism, and fatty acid oxidation genes. Mount 27 contains amino acid metabolism and energy generation genes. Mount 30 contains protein expression genes, and Mount 31 is not characterized. In addition to Mountains, gene expression is also clustered by biofunctional groups. Number of genes in the group represent the number of genes from several experiments that show coregulation of expression for a particular mountain [57].

and significantly up-regulated in lon-2(lf) animals. Of those 20 genes, we find 14 are also highly regulated in our screen (Additional file 5). Included in this subset are the DBL-1 receptor gene, sma-6, and lon-1, a downstream transcriptional target of DBL-1 signaling [7,35]. Our microarray data for lon-1 indicates there may be regulation, similar to what was previously reported, but variation between the data sets puts this result below the $95 \%$ confidence. In our previous study of lon-1, we reported a difference in expression of LON-1 protein between lon-1 and sma-6 of about 30\% [35]. This level of change would not be detected with confidence in a microarray experiment.
Taken together these results suggest that the ultimate effects of DBL-1 signaling on body size in C. elegans may be accomplished through changes in regulating a broad range of genes involved in metabolism and structure.

\section{Hedgehog superfamily signaling is a downstream regulator of DBL-1 signaling}

We identified three hypodermal wrt genes and patched receptor genes in our array. $w r t-1$ and $w r t-8$ were significantly up-regulated, 10 -fold and 8 -fold respectively. wrt-4 was up-regulated but just below statistical significance in our experiments, but was up-regulated 
significantly in Liang et al (2007), using a different set of TGF $\beta$ transducers [37]. Nematodes do not have hedgehog genes, but bioinformatic analysis shows there are several genes that have a conserved Hint domain (autoprocessing domain, similar to the intein domain in hedgehog) but a different $\mathrm{N}$-terminal ligand domain (similar in size to the hedgehog domain, but with no sequence similarity). These genes are called warthog to show their relationship to hedgehog [38-40]. The three warthog genes that are regulated by DBL-1 are exclusively expressed in the hypodermis [38]. All three warthog genes are related to each other in both the wart and Hint domains, with wrt-4 and wrt-8 being most similar to each other ( $\sim 55 \%$ identity between wrt- 4 and wrt- 8 and about $30 \%$ identity between wrt- 1 and the other two).

We obtained gene knockouts from the nematode genome consortium (National BioResource Project; http:// www.shigen.nig.ac.jp/c.elegans/index.jsp) for the three wrt genes in order to test the hypothesis that they affect body size. We made and measured double and triple mutant combinations of animals. Measurements of the wrt mutants singly and in combination with each other show that they are smaller than wild-type animals ( $89 \%$, see Table 3$)$. The double and triple mutant combinations of these three genes do not show a further reduction of body size, suggesting that all three operate in the same pathway. The partial reduction in size observed with the triple mutant, compared to loss of $d b l-1$ pathway function, could be explained by the existence of several other warthog genes that show low levels of expression in the hypodermis [37,38]. A Sma body size for the wrt genes has also been reported in RNAi experiments [41]. Additional evidence that these genes are linked to body size comes from our injection experiments. Overexpression of $w r t-1$ is mostly lethal,

Table 3 Body lengths of warthog mutants.

\begin{tabular}{lccc}
\hline Genotype & $\begin{array}{c}\text { \% length of } \\
\text { N2 }\end{array}$ & $\mathbf{n}$ & P-value \\
\hline wild type (N2) & $100 \pm 2$ & 10 & \\
\hline sma-6(wk7) & $66 \pm 4$ & 21 & $<.001$ \\
\hline wrt-1(tm1417) & $89 \pm 4$ & 17 & 0.290 \\
\hline wrt-4(tm1911) & $89 \pm 4$ & 18 & 0.014 \\
\hline wrt-8(tm1585) & $87 \pm 4$ & 20 & 0.080 \\
\hline wrt-1(tm1417); wrt-4(tm1911) & $85 \pm 3$ & 17 & 0.020 \\
\hline wrt-1(tm1417); wrt-8(tm1585 & $89 \pm 4$ & 12 & 0.036 \\
\hline wrt-8(tm1585); wrt-4(tm1911) & $85 \pm 4$ & 21 & 0.061 \\
\hline wrt-1(tm1417); wrt-8(tm1585); & $87 \pm 4$ & 17 & 0.036 \\
wrt-4(tm1911) & & & \\
\hline Body sie measremt of sng
\end{tabular}

Body size measurements of single, double, and triple mutant combinations. $\mathrm{n}$ represents number of animals measured 48 hours after the L4 stage. The

P-value is the probability that the tested strain length is the same as the wild type. but animals that escaped this terminal phenotype are Sma. Likewise, a partial genomic fragment of wrt-1 fused to GFP is also mostly lethal, with escapers presenting a Sma phenotype. A genomic wrt-8:gfp fusion (containing part of the ligand domain fused to $g f p$, driven by $2180 \mathrm{bp}$ of $w r t-8$ promoter sequence) was injected and the resulting transgenic animals are Sma. These overexpression phenotypes suggest that proper levels of WRT-1 and WRT-8 are required for normal body size morphology.

Liang et al (2007) compared expression patterns between $d b l-1(l f)$, sma-9(lf), and wild-type animals [37]. sma-9 encodes a predicted co-transcription factor for the DBL-1 pathway Smads [42]. They found 31 genes are commonly regulated by sma-9 and $d b l-1$. Only one, $w r t-1$, is down-regulated in both $d b l-1(l f)$ and sma-9(lf) microarrays relative to the wild type. This supports our microarray results showing significant up-regulation of wrt-1 when $d b l-1$ is overexpressed.

While DBL-1 affects the body size of animals living in reproductively favorable conditions, C. elegans has another BMP superfamily member, DAF-7, that regulates an alternative life stage called dauer, a facultative diapause that animals enter in response to harsh environmental conditions $[43,44]$. DBL- 1 and DAF-7 use the same Type II receptor, DAF-4. We reasoned that DBL-1 and DAF-7 might use similar but distinct mechanisms or signaling pathways to regulate their distinct effects. To address this, we compared our results to those from a microarray experiment that compared non-dauer larvae at around the L2 molt to same stage (L2d) animals entering dauer due to loss of function of DAF-7 or the DAF-7 Smads DAF-8 and DAF-14 [45]. The dauer analysis showed that $d b l-1$ is down-regulated in dauering animals, and also identifies several genes related to Hedgehog (Hh) by a common Hog domain, as well as Patched (Hh receptor) genes. Consistent with the downregulation of $d b l-1$ in animals entering dauer, they also found that wrt-1 and wrt-8 were also down-regulated. Seven patched genes were also significantly down-regulated in the dauer study, while we found another, ptr-24, to be 1.3-fold ( $\mathrm{P}=0.014)$ up-regulated. This indicates that DBL-1 and DAF-7 are using similar mechanisms (wrt signaling pathways) to regulate distinct biological outcomes.

\section{Regulation of male spicule development}

Because we used a hermaphrodite population in our studies, we expected to exclude most genes highly regulated by DBL-1 during male tail development. That is largely the case, since most are expressed male-specifically. One that was identified, lin-31, also has roles in hermaphrodite development [46]. LIN-31 is a forkhead transcription factor, which in other systems is a Smad 
co-factor [9]. LIN-31 is implicated in DBL-1-mediated male tail development, as lin-31 mutant males have crumpled spicules like those exhibited by $d b l-1$ pathway mutant males [9]. We show a transcriptional effect of DBL-1 on lin-31, as it is 1.5 -fold $(\mathrm{P}=0.036)$ up-regulated by pathway signaling. This indirectly supports the hypothesis that DBL-1 acts through LIN-31 in affecting spicule development.

\section{Regulation of immunity}

TGF $\beta$ superfamily members play a role in immune responses in mammals [47]. DBL-1 is up-regulated in microarrays analyzing C. elegans innate immunity, and $d b l-1(l f)$ animals succumb sooner than the wild type to infection by pathogenic bacteria and yeast $[5,6,48,49]$. While the DBL-1 pathway is required solely in the hypodermis for its body size role, all receptors and Smads are more strongly expressed in the intestine and/or pharynx, primary sites for the C. elegans immune response [50]. A plausible explanation for DBL-1 pathway expression in the intestine is that it transcriptionally regulates genes required for an immune response. In our microarray study, animal populations were bleached to not only stage them but also to control for possible contamination responses unrelated to genotype. We identified several families of genes known to be involved in the immune response, including lysozymes, lectins, and lipase, as well as $n p r-1$ (Additional file 5) $[5,11,12]$. Other genes with intestinal expression are also enriched (Table 2).

When we compared our results to data obtained from two other microarray analyses analyzing immune response to pathogenic bacterial infection, we identified a remarkable overlap between their highly regulated genes and a subset of ours [5,11]. Mallo et al. analyzed the C. elegans transcriptional response to S. marcescens infection [5]. They identified seven genes with an induction of greater than 2-fold, including a lipase, lectins, and lysozymes, which are involved in immune responses in other animals $[5,11]$. Of those seven, three were identified in our screen as highly up-regulated (Additional file 5). They also found that all of the lysozyme genes they tested (lys-1, -7, and -8) were induced in infected animals by microarray and by northern analyses. We found that these three lys genes were also highly upregulated in our microarray, as were lys-2 and lys-9, which were not represented by cDNAs in the previous study. Wong et al. also identified lipase, lectin, and lysozyme gene up-regulation when they compared animals fed on standard OP50 E. coli to pathogenic E. caratovora, E. faecalis, and P. luminescens-fed animals [11]. They also identified aspartyl proteases and saposin as highly up-regulated. These were identified in our analysis as highly up-regulated (Additional file 5). A directed analysis of immune response in C. elegans by Alper et al. demonstrated DBL- 1 regulation of lys $-1,-7$, and -8 as well as the lectin clec-85 [12].

\section{Development of a DBL-1 pathway fluorescent reporter}

To create a reporter for DBL-1 signaling, we tested six of the highest regulated genes for efficacy as a reporter for the Sma/Mab pathway (T25C12.2, T09F5.9, F35C5.9, Y38E10A.5, W09B7.2, T10H10.2, F11A6.2). We drove green fluorescent protein (GFP) expression from these genes' promoters and compared GFP expression levels in wild-type and sma-6(wk7) animals. We showed that the promoter for an immune-response gene, spp-9/saposin (T25C12.2) showed the greatest difference in response to altered DBL-1 pathway levels $(0.1$-fold regulated, $\mathrm{P}=$ $0.002)[51,52]$. GFP from the spp-9 promoter is weakly expressed in the intestine of wild-type, OP50-fed animals (Fig. 1). However, in the sma-6(wk7) background, this promoter is up-regulated, as seen by increased intestinal fluorescence (Fig. 1). This marker exhibited no change when placed in the background of collagen mutants, which affect body size independently of DBL-1 (data not shown). There are a number of putative Smad binding sites in the promoter region of $s p p-9$, which suggests it may be a direct target, but binding to these sites has not been validated. This strain helps validate our mutants identified from genetic screens, but also provides a screenable marker for future studies.

\section{Conclusions}

Our results show how DBL-1 pathway signaling in the hypodermis leads to body size changes by regulating transcription of genes involved in metabolism, protein synthesis and degradation, but not significantly by cell cycle genes. We identified a proposed downstream signal transduction pathway in the Hh-related wrt signaling pathway, which may relay the DBL-1 pathway signal out of the hypodermis to neighboring cells to regulate body size. We have discovered a mechanism for DBL-1 in the intestinal innate immune response: to promote transcription of many genes directly involved in immunity. A fluorescent biomarker for DBL-1 pathway signaling was generated and will provide the basis for future studies of how DBL-1 signaling is regulated.

\section{Methods}

\section{C. elegans strains}

C. elegans strains were cultured using standard methods at $20^{\circ} \mathrm{C}$ [53]. All mutants used in this paper were derived from the wild-type Bristol strain N2. wrt-1 (tm1417), wrt-4 (tm1911), and wrt-8(tm1585) were isolated by the National BioResource Project http://www. shigen.nig.ac.jp/c.elegans/index.jsp. $r r f-3(p k 1426)$ is described in [54]. wrt-1(tm1417) is created by a deletion 

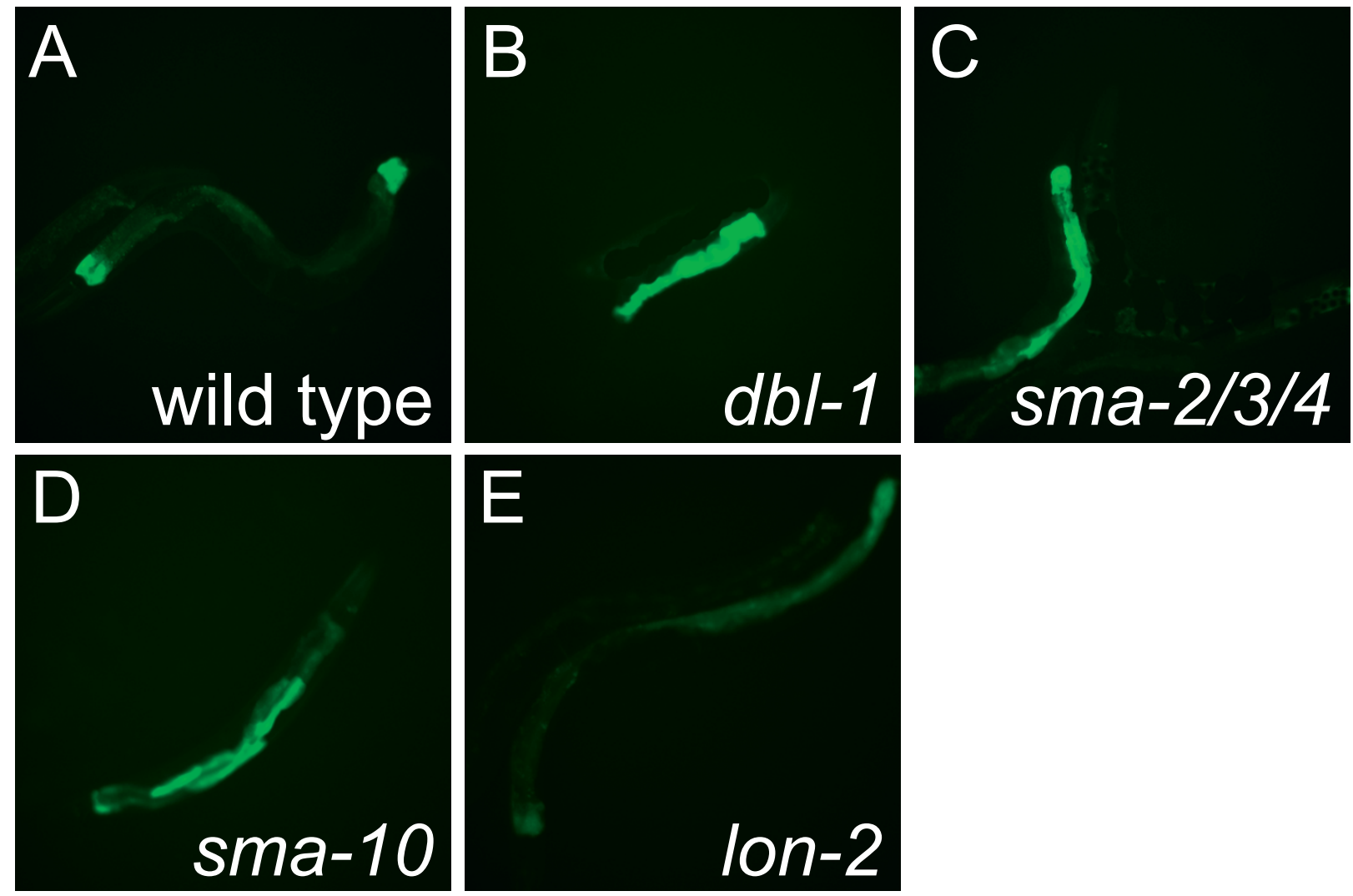

Figure 1 Expression of the SPP-9P::GFP transcriptional reporter in Sma/Mab pathway mutants. The SPP-9P::GFP reporter was crossed into various Sma/Mab pathway mutants to determine the effect of the mutation on the expression level of the reporter. All exposures were of equal duration. A) Repression of the SPP-9P::GFP reporter in a wild-type animal shows little expression in the central region of the intestine, B) SPP-9P:: GFP expression in a $d b l-1, C)$ the triple mutant sma-2,3,4, D) sma-10 mutants show strong expression throughout the center of the intestine, and E) SPP-9P::GFP expression in a lon-2 mutant that overexpresses the pathway shows little expression, similar to the reporter repression seen in wild-type animals.

that removes 616 bp DNA, encoding a protein truncated after amino acid 116 with a short (15 amino acid) missense transcript thereafter. This transcript removes the C-terminal portion of the wrt-1 Wart domain and its Hog domain. wrt-8(tm1585) is caused by a $1256 \mathrm{bp}$ deletion and encodes a protein truncated after 32 amino acids with four additional amino acids of missense transcript. This removes most of the wrt- 8 Wart domain. $w r t-4(t m 1911)$ is a 912 bp deletion that removes exons 4 and 5. Strain LT186 contains a molecular null of the receptor gene, sma-6(wk7) [7]. BW1940 is a strain that contains an integrated $d b l$-1-overexpressing transgene ctIs40 (ZC421 cosmid + pTG96 (sur-5::gfp)) [4]. Microinjection of DNAs into the gonad syncytia of C. elegans hermaphrodites to create transgenic animals was performed by standard microinjection procedures $[55,56]$ and resulted in wkEx52 [spp-9p::gfp], wkEx65 [wrt-8p:: partial wrt-8:gfp + pRF4 (rol-6(su1006))] and $w k E x 66$ [wrt-1p::gfp + pRF4(rol-6(su1006))]. Expression of pRF4 was used to select for transgenic animals.

\section{RNA isolation}

A large population of animals was bleached for eggs. The eggs were then allowed to hatch overnight in M9 media without food in order to synchronize the population at L1. Animals were then plated to NGM plates containing OP50 E. coli. Animals were scored visually for the L4 stage and washed off plates using M9 or 0.1 $\mathrm{M} \mathrm{NaCl}$ solution, then pelleted and dissolved with TRI$\mathrm{zol}^{\odot}$ reagent (Invitrogen Life Technologies, Gaithersburg, MD). We chose the L4 stage to analyze because body length differences are apparent at this stage, it is easy to stage multiple populations at this developmental age, and there will be no possible confounding of results by developing embryos, which are present in adults. After several rounds of vortexing and freeze thaw cycles using liquid nitrogen, the solution was extracted using chloroform, leaving an aqueous solution containing the RNA. The RNA was precipitated using isopropanol, and the pellet was then purified using the $\mathrm{RNeasy}^{\circ}$ kit (Qiagen Inc., Valencia, CA). 


\section{Preparation of Labeled Copy RNA}

Total RNA was extracted from each sample and prepared for hybridization according to the Affymetrix GeneChip Expression Analysis Technical Manual (Affymetrix, 2001). Briefly, RNA was extracted from frozen tissue using the RNeasy ${ }^{\circ}$ Mini kit (Qiagen Inc, Valencia, CA). Sample was further purified and concentrated with an RNeasy MinElute Cleanup column (Qiagen Inc, Valencia, CA). A $200 \mathrm{ng}$ aliquot of each RNA sample was loaded in an RNA 6000 Nano Chip and run on a Bioanalyzer (Agilent Technologies, Palo Alto, CA). The Nano Chip separates the sample via capillary electrophoresis (Agilent Technologies, Palo Alto, CA), and the quality of each sample was determined by evaluating the relative amounts of $28 \mathrm{~S}$ and $18 \mathrm{~S}$ ribosomal peaks.

Five mg of total RNA was used as a template for complementary DNA (cDNA) synthesis with the Superscript Choice System kit (Invitrogen Life Technologies, Gaithersburg, MD). First strand synthesis was primed with a T7-(dT) ${ }_{24}$ oligonucleotide primer containing a T7 RNA polymerase promoter sequence on the 5' end (Genset Oligos, La Jolla, CA). Second strand products were cleaned with the GeneChip ${ }^{\circ}$ Sample Cleanup Module (Affymetrix, CA) and used as a template for in vitro transcription (IVT) with biotin-labeled nucleotides (Bioarray High Yield RNA Transcript Labeling Kit (Enzo Diagnostics, Farmindale, NY). $20 \mathrm{mg}$ of the product was heated at $94^{\circ} \mathrm{C}$ for 35 minutes in fragmentation buffer provided with the Cleanup Module (Affymetrix) in order to produce fragments that were 35-200 base pairs in length.

\section{Array Hybridization}

Fragmented samples were submitted to the University of Florida's joint Shands Cancer Center/Interdisciplinary Center for Biotechnology Research (ICBR) Microarray Core Facility (Gainesville, FL). A $15 \mu \mathrm{g}$ aliquot of fragmented cRNA was hybridized for $16 \mathrm{hr}$ at $45^{\circ} \mathrm{C}$ to an Affymetrix C. elegans GeneChip ${ }^{\circ}$. After hybridization, each array was stained with a streptavidin-phycoerythrin conjugate, washed (Molecular Probes, Eugene, Oregon), and visualized with a GeneArray ${ }^{\text {tm }}$ scanner (Agilent Technologies, Palo Alto, CA). Images were inspected visually for hybridization artifacts. In addition, quality assessment metrics were generated for each scanned image. Microarray core facility staff evaluated these metrics based empirical data from pervious hybridizations and on the signal intensity of internal standards that were present in the hybridization cocktail. Samples that did not pass quality assessment were eliminated from further analyses.

\section{Generation of Expression Values}

Microarray Suite Version 5 software (Affymetrix, Santa Clara, CA) was used to generate .cel files. Probe
Profiler $^{\text {rw }}$ software (v1.3.11) (Corimbia Inc, Berkeley, CA) was used to convert .cel file intensity data into quantitative estimates of gene expression. All expression values were globally scaled to 100 using Probe Profiler ${ }^{\text {rw }}$ software that was developed specifically for the Affymetrix GeneChip system. The software identified informative probe pairs, and down-weighted the signal value of probe pairs that were subject to differential cross-hybridization effects or that consistently produced no signal. The software also detected and corrected for saturation artifacts, outliers and chip defects.

In addition to expression levels, Probe Profiler ${ }^{\text {Twm }}$ generated a probability level associated with the genes' presence or absence. Genes not expressed in at least 2 of the 11 samples $(\mathrm{p}<.05)$ (BW1940: $\mathrm{n}=5$ and LT186: $\mathrm{n}=6$ ) were considered absent. Absent genes were removed from the data set and not included in further analyses.

\section{Data Analysis}

A modified $t$-test was performed on the gene expression values (BW1940: $n=5$ and LT186: $n=6$ ) with Probe Profiler $^{\mathrm{rm}}($ Corimbia Inc., Berkeley, CA). For each analysis, the genes that had a significant treatment effect $(\mathrm{p}=$ $0.05,0.01$ or 0.001 ) were identified. The expression values of these genes were normalized on a gene-bygene basis by first subtracting from each expression value the mean expression value across all arrays, and then dividing standard deviation of values for that gene. In this way a distribution with mean 0 and standard deviation 1 was created for each gene. Hierarchical clustering, K-Means clustering and PCA was performed on the normalized data and visualized with Gene Linker Gold software. All filtering and normalization was performed with AnalyzeIt Tools, a software package developed by the Interdisciplinary Center for Biotechnology Research (ICBR) at the University of Florida.

\section{Array Data Submission}

Array data has been submitted to the Gene Expression Omnibus with accession number GSE15527 http://www. ncbi.nlm.nih.gov/geo/.

\section{CDNA and qRT-PCR}

cDNA libraries were constructed from the RNA of BW1940 ctIs40 and LT186 sma-6(wk7) strains using the iScript cDNA synthesis kit (BioRad). SYBR Green PCR reactions were carried out using a Rotor-Gene RG3000 and the IQ SYBR Green supermix (BioRad). Genes of interest were amplified. A standard curve was used to determine accurate comparisons of transcription levels. Each experimental transcript was compared to an internal control (T11G6.1, histidyl-tRNA synthetase), which showed no significant deviation in our microarray data, in order to obtain a relative expression value. Values 
from three replicates were averaged to determine the overall level of transcription (Table 1).

\section{Body length measurements}

Animals were picked at the L4 stage and photographed as young adults around 48 hours later. Images from individual animals were captured from dissecting microscopes using a QImaging Retiga 1300 cooled color digital camera system and QCapture2 software (Quantitative Imaging Corporation, Burnaby, Canada). Lengths of animals were determined by using Image-Pro Plus measurement software (Media Cybernetics, Inc., Silver Spring, MD).

\section{Reporter Constructs}

Reporter constructs were generated using approximately $3 \mathrm{~kb}$ of DNA upstream of the gene of interest. These promoter sequences were amplified by PCR and cloned into the GFP vector pPD95.75 [55]. After sequencing to verify cloning, the plasmids were injected with marker pRF4 into N2 wild-type young adult hermaphrodites using standard DNA transformation techniques $[55,56]$. Transformed F1 animals were isolated and lines were obtained from transgenic F2 progeny.

\section{Additional material}

Additional file 1: Data Summary of regulated genes. The number of regulated genes scored at confidence intervals of $95 \%, 99 \%$ and $99.9 \%$.

Additional file 2: Genes highly regulated at the $95 \%$ confidence interval. The $99.9 \%$ most highly up- and down-regulated are annotated according to WormBase Release WS211. Other annotations primarily come from the Affymetrix microarray spreadsheet. Some manual crossreferencing was required from the Dauer Metabolic Database http:// dauerdb.org/ to correlate labels from the microarray with WormBase Release WS211.

Additional file 3: Protein synthesis and degradation genes highly regulated at $\mathbf{9 5 \%}$ confidence or above. A summary list of protein synthesis and degradation genes regulated by the Sma/Mab pathway at the $95 \%$ confidence level.

Additional file 4: Structural Genes highly regulated at $95 \%$ confidence or above. A summary list of structural genes regulated by the Sma/Mab pathway at the $95 \%$ confidence level.

Additional file 5: Comparisons with similar microarray experiments. A summary list of comparisons among similar microarray experiments. Mochii et al refers to reference [36], Liang et al refers to reference [37], and Mallo et al refers to reference [5].

\section{List of abbreviations}

BMP: bone morphogenetic protein; L2d: second larval stage entering dauer; L4: fourth larval stage; (If): loss of gene function; IVT: in vitro transcription; TGF $\beta$ : Transforming Growth Factor-beta superfamily.

\section{Acknowledgements}

We thank S. Mitani of the National Biosource Project for the generation of wrt-1, wrt-4, and wrt-8 mutant strains and to the Kim lab and Stanford University for creating web-based tools for data analysis. Some strains were provided by the Caenorhabditis Genetics Center, which is funded by the NIH
National Center for Research Resources. We would like to thank M. Popp and the UFSCC/ICBR Microarray Core for hybridization of the microarrays and advice on statistical analyses, and M. Yang and G. Patterson for assistance in data analysis and preparation. This work was supported by an $\mathrm{NIH}$ grant to R.W.P (HD13465). A.R.F. received partial support by a fellowship from the Charles and Joanna Busch Predoctoral Fellowship and a Biotechnology Training Grant from the NIH. R.J.G. received partial support by a fellowship from the Charles and Joanna Busch Predoctoral Fellowship and from the National Science Foundation Fellowship IGERT \#DGE 0801620. T.L.G. was partially supported by a fellowship from the NIH (AG19957).

\section{Author details}

'Waksman Institute, Department of Molecular Biology and Biochemistry, Cancer Institute of New Jersey, Rutgers University, Piscataway, NJ 088548020, USA. ${ }^{2}$ Current Address: International Life Sciences Institute (ILSI) Research Foundation, Washington D.C. 20005, USA. ${ }^{3}$ Current Address: Department of Molecular and Cellular Medicine, Texas A\&M Health Science Center, College Station, TX 77843-1114, USA.

\section{Authors' contributions}

AFR participated in the design of the experiments, performed the wet lab experiments, assisted with the analysis of the data, and helped draft the manuscript. TLG analyzed the microarray data, performed some genetic experiments, and helped draft the manuscript. RJG participated in image acquisition, analyses of the warthog mutations, and assisted in the preparation of the manuscript. HW participated in the molecular cloning and analyses of molecular constructs. RWP conceived the study, participated in the design and data analyses, and helped draft the manuscript. All authors have read and approved the final manuscript.

Received: 18 August 2009 Accepted: 7 June 2010

Published: 7 June 2010

\section{References}

1. Patterson GI, Padgett RW: TGF $\beta$-related pathways. Roles in Caenorhabditis elegans development. Trends Genet 2000, 16:27-33.

2. Ten Dijke P, Goumans MJ, Itoh F, Itoh S: Regulation of cell proliferation by Smad proteins. J Cell Physiol 2002, 191:1-16.

3. Morita K, Flemming AJ, Sugihara Y, Mochii M, Suzuki Y, Yoshida S, Wood WB, Kohara Y, Leroi AM, Ueno N: A Caenorhabditis elegans TGF- $\beta$, DBL-1, controls the expression of LON-1, a PR-related protein, that regulates polyploidization and body length. Embo J 2002, 21:1063-1073.

4. Suzuki Y, Yandell MD, Roy PJ, Krishna S, Savage-Dunn C, Ross RM, Padgett RW, Wood WB: A BMP homolog acts as a dose-dependent regulator of body size and male tail patterning in Caenorhabditis elegans. Development 1999, 126:241-250.

5. Mallo GV, Kurz CL, Couillault C, Pujol N, Granjeaud S, Kohara Y, Ewbank JJ: Inducible antibacterial defense system in C. elegans. Curr Biol 2002, 12:1209-1214

6. Zugasti O, Ewbank JJ: Neuroimmune regulation of antimicrobial peptide expression by a noncanonical TGF- $\beta$ signaling pathway in Caenorhabditis elegans epidermis. Nat Immunol 2009, 10:249-256.

7. Krishna S, Maduzia LL, Padgett RW: Specificity of TGF $\beta$ signaling is conferred by distinct type I receptors and their associated SMAD proteins in Caenorhabditis elegans. Development 1999, 126:251-260

8. Lints R, Emmons SW: Regulation of sex-specific differentiation and mating behavior in $C$. elegans by a new member of the DM domain transcription factor family. Genes Dev 2002, 16:2390-2402.

9. Baird SE, Ellazar SA: TGFB-like signaling and spicule development in Caenorhabditis elegans. Dev Biol 1999, 212:93-100.

10. Chen W, Chen S, Yap SF, Lim L: The Caenorhabditis elegans p21-activated kinase (CePAK) colocalizes with CeRac1 and CDC42Ce at hypodermal cell boundaries during embryo elongation. I Biol Chem 1996, 271:26362-26368.

11. Wong D, Bazopoulou D, Pujol N, Tavernarakis N, Ewbank JJ: Genome-wide investigation reveals pathogen-specific and shared signatures in the response of Caenorhabditis elegans to infection. Genome Biol 2007, 8:R194.

12. Alper S, McBride SJ, Lackford B, Freedman JH, Schwartz DA: Specificity and complexity of the Caenorhabditis elegans innate immune response. Mol Cell Biol 2007, 27:5544-5553. 
13. Tenor $J$, Aballay A: A conserved Toll-like receptor is required for Caenorhabditis elegans innate immunity. EMBO Rep 2008, 9:103-109.

14. Nurse P: The genetic control of cell volume. The Evolution of Genome Size John Wiley and SonsCavalier-Smith T 1985, 185-196.

15. Conlon I, Raff M: Size control in animal development. Cell 1999, 96:235-244.

16. Gao FB, Raff M: Cell size control and a cell-intrinsic maturation program in proliferating oligodendrocyte precursor cells. J Cell Biol 1997, 138:1367-1377.

17. Guo X, Allen SK Jr: Sex determination and polyploid gigantism in the dwarf surfclam (Mulinia lateralis Say). Genetics 1994, 138:1199-1206.

18. Galitski T, Saldanha AJ, Styles CA, Lander ES, Fink GR: Ploidy regulation of gene expression. Science 1999, 285:251-254

19. Oldham S, Stocker H, Laffargue M, Wittwer F, Wymann M, Hafen E: The Drosophila insulin/IGF receptor controls growth and size by modulating PtdlnsP(3) levels. Development 2002, 129:4103-4109.

20. Savage C, Das P, Finelli AL, Townsend SR, Sun CY, Baird SE, Padgett RW: Caenorhabditis elegans genes sma-2, sma-3, and sma-4 define a conserved family of transforming growth factor- $\beta$ pathway components. Proc Natl Acad Sci USA 1996, 93:790-794.

21. Spencer FA, Hoffmann FM, Gelbart WM: Decapentaplegic: a gene complex affecting morphogenesis in Drosophila melanogaster. Cell 1982, 28:451-461.

22. Jones P, Chase K, Martin A, Davern P, Ostrander EA, Lark KG: Singlenucleotide-polymorphism-based association mapping of dog stereotypes. Genetics 2008, 179:1033-1044.

23. Lee SJ: Regulation of muscle mass by myostatin. Annu Rev Cell Dev Biol 2004, 20:61-86

24. Massague J: TGFß in Cancer. Cell 2008, 134:215-230.

25. Sulston JE, Horvitz HR: Post-embryonic cell lineages of the nematode, Caenorhabditis elegans. Dev Biol 1977, 56:110-156.

26. Jorgensen P, Nishikawa JL, Breitkreutz BJ, Tyers M: Systematic identification of pathways that couple cell growth and division in yeast. Science 2002 297:395-400.

27. Lambertsson A: The minute genes in Drosophila and their molecular functions. Adv Genet 1998, 38:69-134.

28. Saeboe-Larssen S, Lyamouri M, Merriam J, Oksvold MP, Lambertsson A: Ribosomal protein insufficiency and the minute syndrome in Drosophila: a dose-response relationship. Genetics 1998, 148:1215-1224.

29. Weijers D, Franke-van Dijk M, Vencken RJ, Quint A, Hooykaas P, Offringa R: An Arabidopsis Minute-like phenotype caused by a semi-dominant mutation in a ribosomal protein S5 gene. Development 2001, 128:4289-4299.

30. Luo S, Shaw WM, Ashraf J, Murphy CT: TGF- $\beta$ Sma/Mab signaling mutations uncouple reproductive aging from somatic aging. PLoS Genet 2009, 5:e1000789.

31. Nystrom J, Shen ZZ, Aili M, Flemming AJ, Leroi A, Tuck S: Increased or decreased levels of Caenorhabditis elegans lon-3, a gene encoding a collagen, cause reciprocal changes in body length. Genetics 2002, 161:83-97.

32. Affolter M, Basler K: The Decapentaplegic morphogen gradient: from pattern formation to growth regulation. Nat Rev Genet 2007, 8:663-674.

33. Lozano E, Saez AG, Flemming AJ, Cunha A, Leroi AM: Regulation of growth by ploidy in Caenorhabditis elegans. Curr Biol 2006, 16:493-498.

34. Flemming AJ, Shen ZZ, Cunha A, Emmons SW, Leroi AM: Somatic polyploidization and cellular proliferation drive body size evolution in nematodes. Proc Natl Acad Sci USA 2000, 97:5285-5290.

35. Maduzia LL, Gumienny TL, Zimmerman CM, Wang H, Shetgiri P, Krishna S, Roberts AF, Padgett RW: Ion-1 regulates Caenorhabditis elegans body size downstream of the $d b /$-1/TGF $\beta$ signaling pathway. Dev Biol 2002, 246:418-28.

36. Mochii M, Yoshida S, Morita K, Kohara Y, Ueno N: Identification of transforming growth factor- $\beta$-regulated genes in Caenorhabditis elegans by differential hybridization of arrayed cDNAs. Proc Natl Acad Sci USA 1999, 96:15020-15025.

37. Liang J, Yu L, Yin J, Savage-Dunn C: Transcriptional repressor and activator activities of SMA-9 contribute differentially to BMP-related signaling outputs. Dev Biol 2007, 305:714-725.

38. Aspock G, Kagoshima H, Niklaus G, Burglin TR: Caenorhabditis elegans has scores of hedgehog-related genes: sequence and expression analysis. Genome Res 1999, 9:909-923.
39. Burglin TR: Warthog and groundhog, novel families related to hedgehog. Curr Biol 1996, 6:1047-1050

40. Porter JA, Ekker SC, Park WJ, von Kessler DP, Young KE, Chen CH, Ma Y, Woods AS, Cotter RJ, Koonin EV, et al: Hedgehog patterning activity: role of a lipophilic modification mediated by the carboxy-terminal autoprocessing domain. Cell 1996, 86:21-34

41. Zugasti O, Rajan J, Kuwabara PE: The function and expansion of the Patched- and Hedgehog-related homologs in C. elegans. Genome Res 2005, 15:1402-1410.

42. Liang J, Lints R, Foehr ML, Tokarz R, Yu L, Emmons SW, Liu J, SavageDunn C: The Caenorhabditis elegans schnurri homolog sma-9 mediates stage- and cell type-specific responses to DBL-1/BMP-related signaling. Development 2003, 130:6453-6464.

43. Riddle $\mathrm{DL}$, Swanson MM, Albert PS: Interacting genes in nematode dauer larva formation. Nature 1981, 290:668-671.

44. Hu PJ: Dauer. The C elegans Research Community WormBook 2007 [http:// wormbook.org], vol. doi/10.1895/wormbook.1.144.1.

45. Liu T, Zimmerman KK, Patterson Gl: Regulation of signaling genes by TGF $\beta$ during entry into dauer diapause in C. elegans. BMC Dev Biol 2004, $4: 11$.

46. Miller LM, Gallegos ME, Morisseau BA, Kim SK: lin-31, a Caenorhabditis elegans HNF-3/fork head transcription factor homolog, specifies three alternative cell fates in vulval development. Genes Dev 1993, 7:933-947.

47. Taylor AW: Review of the activation of TGF- $\beta$ in immunity. J Leukoc Biol 2009, 85:29-33.

48. Millet AC, Ewbank JJ: Immunity in Caenorhabditis elegans. Curr Opin Immunol 2004, 16:4-9.

49. Schulenburg $H$, Kurz $C L$, Ewbank JJ: Evolution of the innate immune system: the worm perspective. Immunol Rev 2004, 198:36-58.

50. McGhee JD: The C. elegans intestine. The C elegans Research Community WormBook 2007 [http://wormbook.org], vol. doi/10.1895/wormbook.1.133.1.

51. Nicholas HR, Hodgkin J: Responses to infection and possible recognition strategies in the innate immune system of Caenorhabditis elegans. Mol Immunol 2004, 41:479-493.

52. Winau F, Schwierzeck V, Hurwitz R, Remmel N, Sieling PA, Modlin RL, Porcelli SA, Brinkmann V, Sugita M, Sandhoff $K$, et al: Saposin C is required for lipid presentation by human CD1b. Nat Immunol 2004, 5:169-174.

53. Brenner S: The genetics of Caenorhabditis elegans. Genetics 1974, 77:71-94.

54. Duchaine TF, Wohlschlegel JA, Kennedy S, Bei Y, Conte D Jr, Pang K, Brownell DR, Harding S, Mitani S, Ruvkun G, et al: Functional proteomics reveals the biochemical niche of $C$. elegans DCR-1 in multiple smallRNA-mediated pathways. Cell 2006, 124:343-354.

55. Mello C, Fire A: DNA Transformation. Caenorhabditis elegans: Modern Biological Analysis of an Organism San Diego, California: Academic Press IncEpstein HF, Shakes DC 1995, 48:451-482.

56. Mello CC, Kramer JM, Stinchcomb D, Ambros V: Efficient gene transfer in $C$. elegans: extrachromosomal maintenance and integration of transforming sequences. Embo J 1991, 10:3959-3970.

57. Kim SK, Lund J, Kiraly M, Duke K, Jiang M, Stuart JM, Eizinger A, Wylie BN, Davidson GS: A gene expression map for Caenorhabditis elegans. Science 2001, 293:2087-2092.

doi:10.1186/1471-213X-10-61

Cite this article as: Roberts et al:: Regulation of genes affecting body

size and innate immunity by the DBL-1/BMP-like pathway in

Caenorhabditis elegans. BMC Developmental Biology 2010 10:61. 